\title{
Bipolar Conduction as the Possible Origin of the Electronic Transition in Pentatellurides: Metallic vs Semiconducting Behavior
}

\author{
P. Shahi, ${ }^{1,2}$ D. J. Singh, ${ }^{3,}$ J. P. Sun, ${ }^{1,2}$ L. X. Zhao, ${ }^{1,2}$ G. F. Chen, ${ }^{1,2}$ Y. Y. Lv, ${ }^{4}$ \\ J. Li, ${ }^{4}$ J.-Q. Yan, ${ }^{5}$ D. G. Mandrus, ${ }^{5,6}$ and J.-G. Cheng ${ }^{1,2, \dagger}$ \\ ${ }^{1}$ Beijing National Laboratory for Condensed Matter Physics and Institute of Physics, \\ Chinese Academy of Sciences, Beijing 100190, China \\ ${ }^{2}$ School of Physical Sciences, University of Chinese Academy of Sciences, Beijing 100190, China \\ ${ }^{3}$ Department of Physics and Astronomy, University of Missouri, Columbia, Missouri 65211-7010, USA \\ ${ }^{4}$ Research Institute of Superconductor Electronics, Nanjing University, Nanjing 210093, China \\ ${ }^{5}$ Materials Science and Technology Division, Oak Ridge National Laboratory, \\ Oak Ridge, Tennessee 37831, USA \\ ${ }^{6}$ Department of Materials Science and Engineering, University of Tennessee, \\ Knoxville, Tennessee 37996, USA
}

(Received 6 February 2018; revised manuscript received 12 April 2018; published 30 May 2018)

\begin{abstract}
The pentatellurides $\mathrm{ZrTe}_{5}$ and $\mathrm{HfTe}_{5}$ are layered compounds with one-dimensional transition-metal chains that show a not-yet-understood temperature-dependent transition in transport properties as well as recently discovered properties suggesting topological semimetallic behavior. Here, we report magnetotransport properties for two kinds of $\mathrm{ZrTe}_{5}$ single crystals grown with the chemical vapor transport (CVT) and the flux method (Flux), respectively. They show distinct transport properties at zero field: The CVT crystal displays a metallic behavior with a pronounced resistance peak and a sudden sign reversal in thermopower at approximately $130 \mathrm{~K}$, consistent with previous observations of the electronic transition; in striking contrast, the Flux crystal exhibits a semiconducting-like behavior at low temperatures and a positive thermopower over the whole temperature range. For both samples, strong effects on the transport properties are observed when the magnetic field is applied along the orthorhombic $b$ and $c$ axes, i.e., perpendicular to the chain direction. Refinements on the single-crystal x-ray diffraction and the measurements of energy dispersive spectroscopy reveal the presence of noticeable Te vacancies in the CVT samples, while the Flux samples are close to the stoichiometry. Analyses on the magnetotransport properties confirm that the carrier densities of the CVT sample are about two orders higher than those of the Flux sample. Our results thus indicate that the widely observed anomalous transport behaviors in pentatellurides actually take place in the Te-deficient samples. For the stoichiometric pentatellurides, our electronic structure calculations show narrow-gap semiconducting behavior, with different transport anisotropies for holes and electrons. For the degenerately doped $n$-type samples, our transport calculations can result in a resistivity peak and crossover in thermopower from negative to positive at temperatures close to those observed experimentally due to a combination of bipolar effects and different anisotropies of electrons and holes. Our present work resolves the long-standing puzzle regarding the anomalous transport behaviors of pentatellurides, as well as the electronic structure in favor of a semiconducting state.
\end{abstract}

DOI: 10.1103/PhysRevX.8.021055

*singhdj@missouri.edu

†jgcheng@iphy.ac.cn

Published by the American Physical Society under the terms of the Creative Commons Attribution 4.0 International license. Further distribution of this work must maintain attribution to the author(s) and the published article's title, journal citation, and DOI.

\author{
Subject Areas: Condensed Matter Physics, \\ Materials Science, \\ Topological Insulators
}

\section{INTRODUCTION}

The pentatellurides $\mathrm{ZrTe}_{5}$ and $\mathrm{HfTe}_{5}$ are layered materials [1,2] with a high concentration of the heavy $p$-element Te. They were found to show a remarkable electronic transition as a function of temperature ( $T$ ) [3]. As depicted in Fig. 1(a), these compounds occur in an orthorhombic space group $\mathrm{Cmcm}$ with layers stacked along the $b$ axis and the chains of $\mathrm{Zr}$ or $\mathrm{Hf}$ atoms running along the $a$ axis. The crystals generally grow with a needlelike or ribbonlike morphology, with the longest 

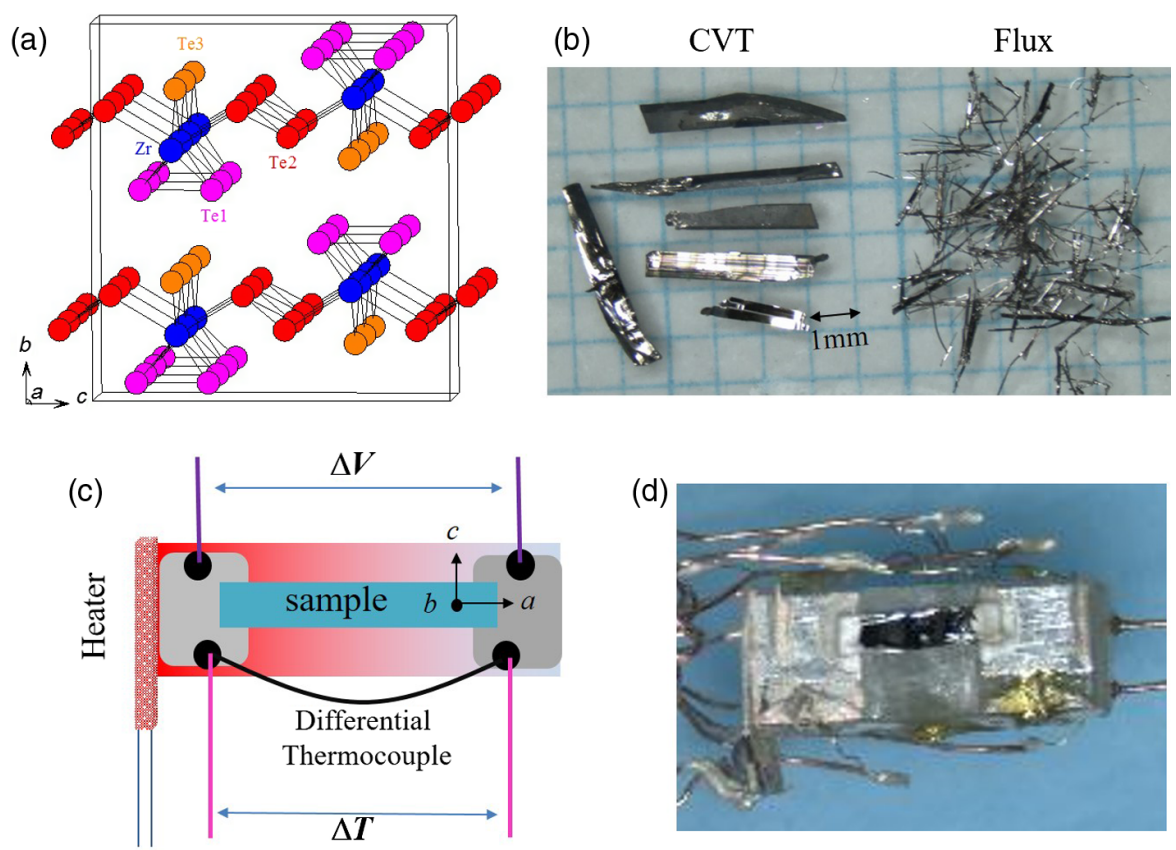

FIG. 1. (a) Crystal structure of $\mathrm{ZrTe}_{5}$. (b) $\mathrm{ZrTe}_{5}$ crystals grown with the CVT and Flux methods. (c) A schematic drawing of the sample stage for thermopower measurements. (d) The thermopower stage with a $\mathrm{ZrTe}_{5}$ crystal on it. This stage can be inserted into the piston-cylinder cell for high-pressure thermopower measurements up to $2.5 \mathrm{GPa}$.

dimension along the chain direction. Transport measurements are thus normally made along the $a$-axis direction. Importantly, both compounds were reported to show a strong peak in resistivity, $\rho(T)$, accompanied by a change in sign of the thermopower, $S(T)$, from electronlike to holelike as $T$ is increased. This takes place at $130-150 \mathrm{~K}$ for $\mathrm{ZrTe}_{5}$ and $50-80 \mathrm{~K}$ for $\mathrm{HfTe}_{5}$ samples both grown by chemical vapor transport technique [3]. It was observed earlier that this transition does not show a sharp structure in resistivity; rather, it has a continuous behavior [3], and there is no evident structural distortion around the transition as in, for example, a charge density wave [4]. It was also noted that these compounds have rather high values of thermopower around the peak, making them of interest as potential thermoelectric materials [5]. Finally, it is noteworthy that samples grown by different methods show qualitatively similar behavior but that the temperature at which the peak occurs may differ. For example, recent flux-grown $\mathrm{ZrTe}_{5}$ samples show a peak at approximately $60 \mathrm{~K}$ [6]. In any case, possible causes for this behavior were advanced. These include density waves [3], inconsistent with the diffraction and high magnetic field data [4]; polaronic models [7], which are, however, apparently inconsistent with the good low-temperature conduction; a semimetal-semiconductor phase transition [8] or a temperature-induced Lifshitz transition [9,10]. More recently, these compounds have become of interest as topological materials whose low-energy electronic structure is controlled by spin-orbit interactions [11]. Nevertheless, it is currently under hot debate whether they are insulators or Dirac semimetals [6,12-18].
In order to resolve these issues, we have performed a comprehensive study of the transport properties of two kinds of $\mathrm{ZrTe}_{5}$ crystals grown with the chemical vapor transport (CVT) method and the flux method, denoted as CVT and Flux hereafter, respectively. These are the two main techniques that have been used to grow pentatelluride single crystals. Our results on the CVT sample agree well with the previous observations, i.e., the metallic behavior with a resistivity peak and a sign reversal of thermopower, but the Flux sample displays semiconducting-like behavior with positive thermopower in the whole temperature range. These distinct behaviors are found to originate from the quite-different $\mathrm{Zr}$ :Te molar ratios, i.e., 1:4.86 for CVT versus 1:4.98 for Flux, based on the refinements of singlecrystal x-ray diffraction data. The presence of Te vacancies in CVT was verified by the energy dispersive spectroscopy measurements. Since the Te vacancies are an expected $n$-type dopant, the carrier density of CVT samples was indeed found to be two orders higher than that of the Flux samples. Electronic structure calculations for stoichiometric material show narrow-gap semiconducting behavior, with a two-dimensional electronic structure having opposite high-conductivity directions for electrons and holes. Transport calculations show that with $n$-type doping, a resistivity peak at temperatures consistent with experiment is obtained. This peak is accompanied by a change in sign from $n$-type to $p$-type thermopower and has its origin in the onset of bipolar conduction. Simple semiconducting behavior is obtained without doping. The effect of the magnetic field can be explained by a standard field-dependent 
reduction of conductivity perpendicular to the field direction. Thus, our present work may resolve the long-standing puzzle regarding the anomalous transport behaviors and also the question of the electronic structure in favor of a semiconducting state.

\section{EXPERIMENTAL DETAILS}

\section{A. Crystal growth}

The $\mathrm{ZrTe}_{5}$ single crystals used in the present study were grown by chemical vapor transport for the CVT samples [16] and by the Te-flux method for the Flux samples [18]. We employed iodine $\left(\mathrm{I}_{2}\right)$ as the transport agent during the CVT crystal growth. $\mathrm{Zr}$ (powder $99.2 \%$, Hf nominal $4.5 \%$ ) and $\mathrm{Te}$ (powder, $5 \mathrm{~N}$ ) were mixed in the molar ratio of $\mathrm{Zr}: \mathrm{Te}=1: 5.5$ and then sealed in a quartz ampoule with $\mathrm{I}_{2}$ $(7 \mathrm{mg} / \mathrm{mL})$. The ampoule was placed in a two-zone furnace. A typical temperature gradient from $480^{\circ} \mathrm{C}$ to $400^{\circ} \mathrm{C}$ was applied. After two months, long ribbon-shaped single crystals were obtained, as shown in Fig. 1(b). On the other hand, we used a Canfield crucible set (CCS) [19] to grow $\mathrm{ZrTe}_{5}$ crystals out of the Te flux [18]. A Zr slug $(99.95 \%$, Hf nominal $3 \%)$ and Te shots $(5 \mathrm{~N})$ in an atomic ratio of $1: 49$ were loaded into the CCS and then sealed in a silica ampoule under vacuum. The sealed ampoule was heated to $1000^{\circ} \mathrm{C}$ and kept for $12 \mathrm{~h}$ to homogenize the melt. It was furnace cooled to $650^{\circ} \mathrm{C}$ and then cooled down to $460^{\circ} \mathrm{C}$ in $60 \mathrm{~h} . \mathrm{ZrTe}_{5}$ crystals were isolated from Te flux by centrifuging at $460^{\circ} \mathrm{C}$. The flux-grown $\mathrm{ZrTe}_{5}$ crystals have a needle shape with typical size of about 1-2 $\mathrm{mm}$ long and $0.01-0.2 \mathrm{~mm}$ in the other two dimensions. As compared in Fig. 1(b), the Flux samples are much narrower than the CVT samples, presumably due to the shorter growth period.

\section{B. Structural and composition measurements}

The composition of these crystals was characterized by the energy-dispersive x-ray spectroscopy (EDS) with an Ametek@EDAX (Model Octane Plus) spectrometer, equipped in a field-emission scanning electron microscope (Hitachi S-4800). Single-crystal x-ray diffraction (XRD) data were collected from a single crystal coated with mineral oil with Mo $K_{\alpha}$ radiation, $\lambda=0.71073 \AA$, in a Bruker D8 Venture Photon II diffractometer equipped with a multifilm monochromator. Structure refinement was carried out with the program SHELXL-2014/7 implemented in the program suite Apex 3.

\section{Electrical transport measurements}

The temperature dependence of resistivity $\rho(T)$ was measured with the standard four-probe method having the current injected along the $a$ axis, the longest dimension of these crystals. The thermopower $S(T)$ measurements were performed by using a homemade setup that was integrated into the commercial Magnetic Property Measurement System (MPMS-3, Quantum Design). Figures 1(c) and 1(d) depict the sample stage used for the thermopower measurements. The temperature gradient $\Delta T / T \sim 1 \%$ was maintained along the $a$ axis during the $S(T)$ measurements and was recorded with a AuFe/chromel differential thermocouple with an accuracy of $0.2 \%$. The precision of the thermopower is about $0.5 \mu \mathrm{V} / \mathrm{K}$. To investigate the effect of magnetic fields on both $\rho(T)$ and $S(T)$, we have applied various magnetic fields up to $7 \mathrm{~T}$ along the three principal crystallographic axes. The effect of magnetic fields on the thermocouple was found to be negligible. Longitudinal magnetoresistance and Hall resistivity were also measured in a Physical Property Measurement System (PPMS-9T, Quantum Design) for both crystals with the current along the $a$ axis and the magnetic field along the $b$ axis.

\section{Density functional calculations}

Our density functional calculations were performed using the general potential linearized augmented planewave (LAPW) method [20] as implemented in the WIEN2k code [21]. We used sphere radii of $2.4 \mathrm{bohr}$ for $\mathrm{Zr}$ and $\mathrm{Hf}$, and $2.5 \mathrm{bohr}$ for $\mathrm{Te}$, along with local orbitals to treat the semicore states. We used the standard LAPW basis with local orbitals [22] rather than the more efficient APW + lo method [23], which has larger errors, especially away from the linearization energies, potentially of importance for the spin-orbit calculation. We used highly converged basis sets, determined by the criterion $R_{\min } k_{\max }=9.0\left(R_{\min }=2.4 \mathrm{bohr}\right.$ is the radius of the smallest sphere and $k_{\max }$ is the planewave sector cutoff), along with dense zone samplings. The calculations were based on the experimental lattice parameters [2], with all internal atomic coordinates relaxed using the PBE exchange correlation functional [24]. For technical reasons, this relaxation was done in a scalar relativistic approximation. We used the resulting crystal structures to calculate the electronic properties and transport coefficients, including spin-orbit interaction. The transport coefficients were obtained using the BoltzTraP code [25]. In the calculations presented, the chemical doping level (i.e., carrier concentration) was held fixed as a function of temperature, as is generally appropriate for semiconductors below the melting point. This leads to a temperature-dependent Fermi energy.

\section{RESULTS AND DISCUSSION}

\section{A. Transport properties at zero magnetic field}

Figure 2 displays the temperature dependence of (a) resistivity $\rho(T)$ and (b) thermopower $S(T)$ in the temperature range $2 \mathrm{~K}<T<300 \mathrm{~K}$ under zero magnetic field for the two different $\mathrm{ZrTe}_{5}$ crystals. The results for CVT and Flux shown here are representative of the single crystals made using different methods and are found to be reproducible by checking several crystals for each method. For the CVT samples, $\rho(T)$ initially decreases slightly and then exhibits a broad peak centered around $T_{p} \approx 132 \mathrm{~K}$, below which a 


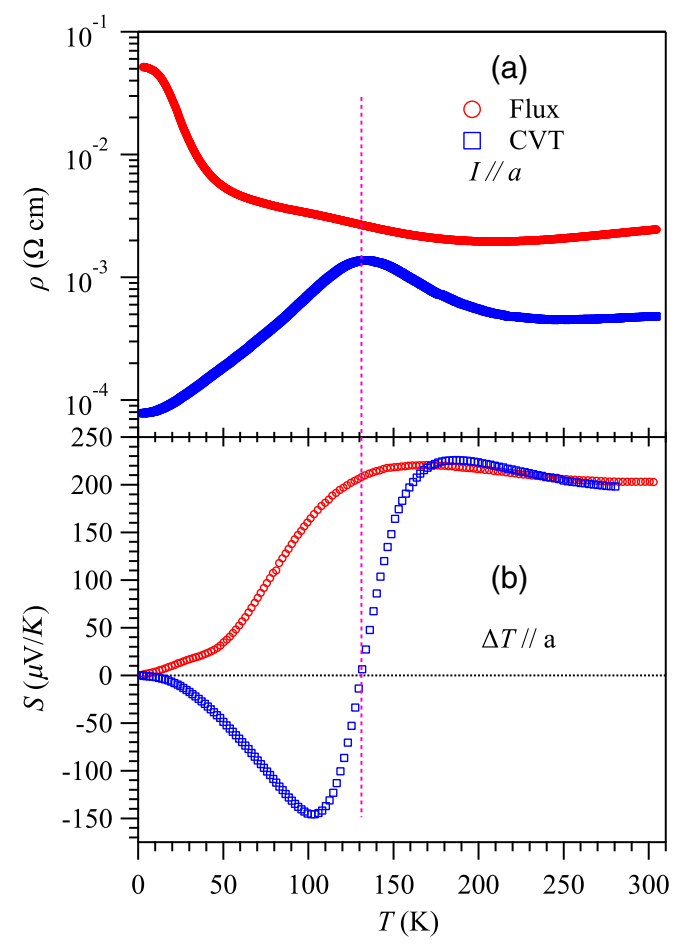

FIG. 2. Temperature dependence of (a) resistivity $\rho(T)$ and (b) thermopower $S(T)$ in zero magnetic field for the $\mathrm{ZrTe}_{5}$ crystals grown with the CVT and Flux methods. The vertical dashed line marks the characteristic temperature $T_{p}$ of CVT sample.

typical metallic behavior was recovered; the thermopower attains a large, positive value of about $200 \mu \mathrm{V} / \mathrm{K}$ at room temperature, and it undergoes a dramatic sign change crossing zero at $T_{p}$ (see the dotted line in Fig. 2). All these observations in CVT are consistent with previously published results $[3,5]$. Surprisingly, we observed totally different transport behaviors for the Flux sample. As seen in Fig. 2, its $\rho(T)$ first decreases upon cooling, showing a metallic behavior, for $200 \mathrm{~K}<T<300 \mathrm{~K}$, but then it shows a semiconducting-like behavior by undergoing two successive upturns at about $150 \mathrm{~K}$ and $50 \mathrm{~K}$, before reaching a plateau at low temperatures. Note that $S(T)$ remains positive in the whole temperature range without any sign change, yet it attains a similar value of about $200 \mu \mathrm{V} / \mathrm{K}$ at room temperature. It is noteworthy that a corresponding slope change of $S(T)$ can be discerned at the characteristic temperatures where the $\rho(T)$ also exhibits anomalies around $150 \mathrm{~K}$ and $50 \mathrm{~K}$.

As mentioned, the resistivity peak and the sign reversal in thermopower at $T_{p} \approx 130 \mathrm{~K}$ in $\mathrm{ZrTe}_{5}$ have been known since 1980s [3], but a proper understanding of these features is lacking. The present study demonstrates that these anomalous transport properties are actually sample dependent, and they appear only in the CVT crystals. The absence of the resistivity peak and sign reversal of thermopower in the Flux crystals is a new observation in the present study. Before we clarify the sample differences for these $\mathrm{ZrTe}_{5}$ crystals and discuss the underlying physics, we first demonstrate similar effects of magnetic fields on the transport properties.

\section{B. Transport properties under magnetic fields}

Despite the apparently different transport properties of these two kinds of $\mathrm{ZrTe}_{5}$ single crystals, a closer inspection of the data in Fig. 2 shows that the characteristic anomalies in $\rho(T)$ and $S(T)$ actually take place at nearly the same temperature for CVT and Flux samples. For example, the Flux sample shows a broad hump feature in $\rho(T)$, together with a downturn trend in $S(T)$ near the $T_{p}$ of the CVT sample. This fact implies that these transport property anomalies might have a common origin that is intrinsic to $\mathrm{ZrTe}_{5}$. The similar effects of magnetic fields on the transport properties shown in Figs. 3 and 4 further elaborate this point.

Figure 3 shows the $\rho(T)$ and $S(T)$ data of the CVT sample under various magnetic fields up to $7 \mathrm{~T}$ applied along the three principal axes. We have plotted the data in the same scale to clearly see the different effects of the magnetic field. As shown in Figs. 3(a) and 3(b), the effect of the magnetic field on $\rho(T)$ and $S(T)$ is negligible when $H / / a$. In contrast, the influence of the magnetic field is much enhanced for $H / / c$; as seen in Figs. 3(c) and 3(d), the most dramatic changes are observed in the temperature range around $T_{p}$, where the magnitudes for both the resistivity peak and the negative thermopower peak are enhanced by a factor of $2-3$. In addition, $T_{p}$ shifts slightly towards a higher temperature with increasing magnetic field as reported previously [26]. The influence of the magnetic field on $\rho(T)$ and $S(T)$ is the most pronounced for $H / / b$, i.e., the direction perpendicular to the $\mathrm{ZrTe}_{5}$ sheets [Fig. 1(a)]. As seen in Figs. 3(e) and 3(f), the resistance peak and the negative thermopower peak become 4-6 times larger under a magnetic field $\mu_{0} H=7 \mathrm{~T}$. It should be noted that both $\rho(T)$ and $S(T)$ exhibit obvious enhancement in a wide temperature range well above $T_{p}$ for this configuration, while the effect is hard to see above $T_{p}$ for $H / / c$.

Similar anisotropic effects of the magnetic field on the transport properties of the Flux sample were also observed. As can be seen in Fig. 4, both $\rho(T)$ and $S(T)$ hardly change under magnetic fields up to $7 \mathrm{~T}$ for $H / / a$, while they are enhanced significantly when the magnetic field is applied along the $b$ and $c$ axes. For $H / / c$, the enhancements appear mainly at temperatures below about $150 \mathrm{~K}$; in particular, pronounced $S(T)$ peaks reaching as high as about $500 \mu \mathrm{V} / \mathrm{K}$, accompanied by wide $\rho(T)$ plateaus, emerged below about $100 \mathrm{~K}$ upon the application of magnetic fields. Although similar features are observed for $H / / b$, the influence of the magnetic field at $T>200 \mathrm{~K}$ is much stronger than that for $H / / c$. Although the field effect is weak for $H / / a$, some unusual features are noticeable at low temperatures in Fig. 4(a). We have thus measured $\rho(H)$ at low temperatures for $H / / I / / a$ and shown the results in Fig. S1 in Supplemental Material [27]. As can be seen, the 

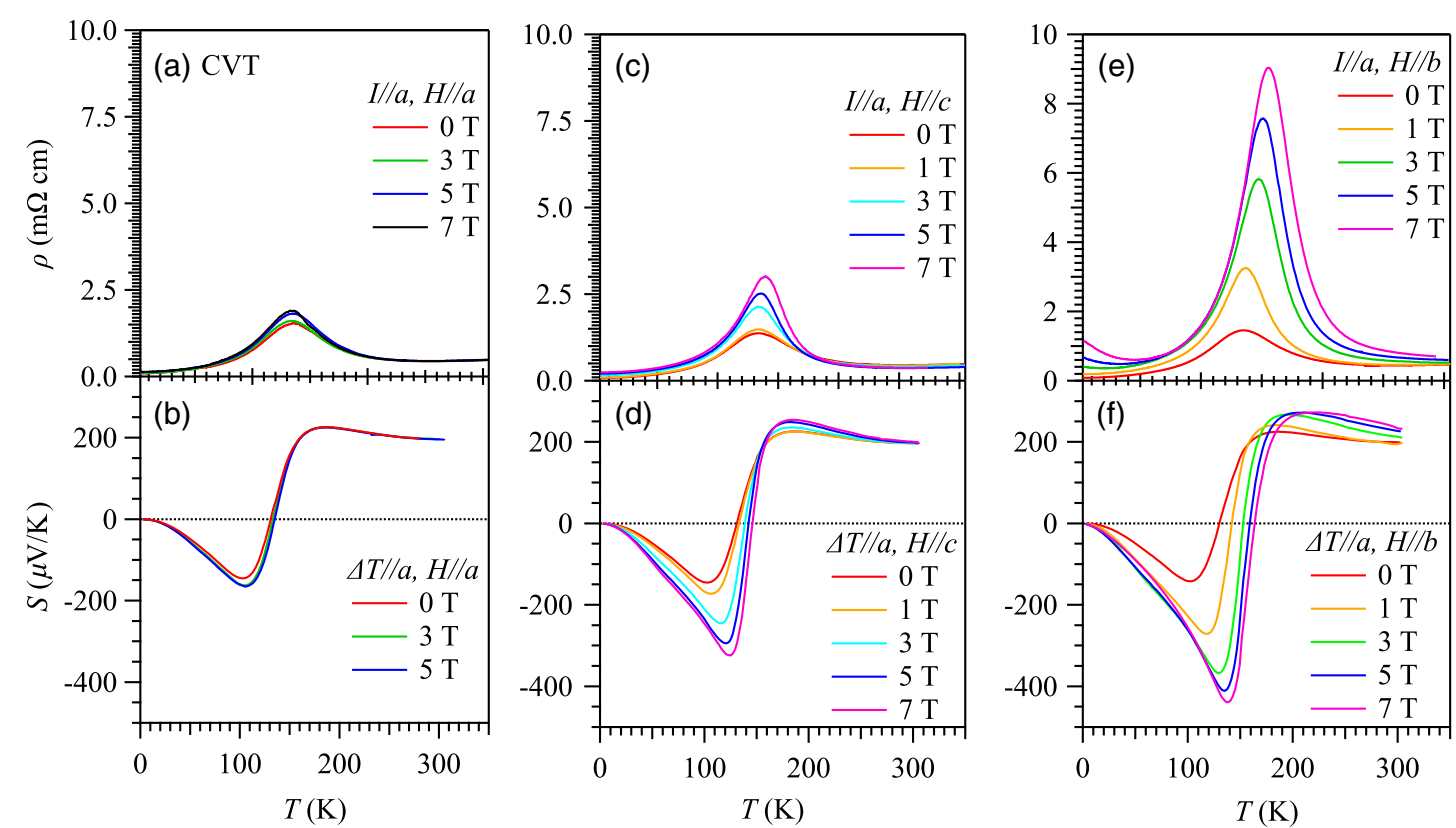

FIG. 3. Temperature dependence of resistivity $\rho(T)$ and thermopower $S(T)$ for the CVT sample with magnetic field applied along the three principal axes.

negative MR becomes prominent for $T \geq 10 \mathrm{~K}$ at $\mu_{0} H>1 \mathrm{~T}$. The results are similar to those reported by Li et al. [12], and they were interpreted in terms of the chiral magnetic effect. However, as far as we know, negative MR might also come from several other extrinsic effects, such as the superimposition of Hall signals, fielddependent inhomogeneous current flow in the bulk, and weak localization of coexistent trivial carriers [28,29].
Therefore, further studies are needed to pin down the origin of the observed negative MR.

From the above characterizations, we can conclude that the influences of the magnetic field on the transport properties of these two kinds of $\mathrm{ZrTe}_{5}$ crystals are quite similar, and the strongest effect appears mainly in the vicinity of the characteristic anomalies, regardless of the detailed difference of band fillings. Given the 2D character of the crystal
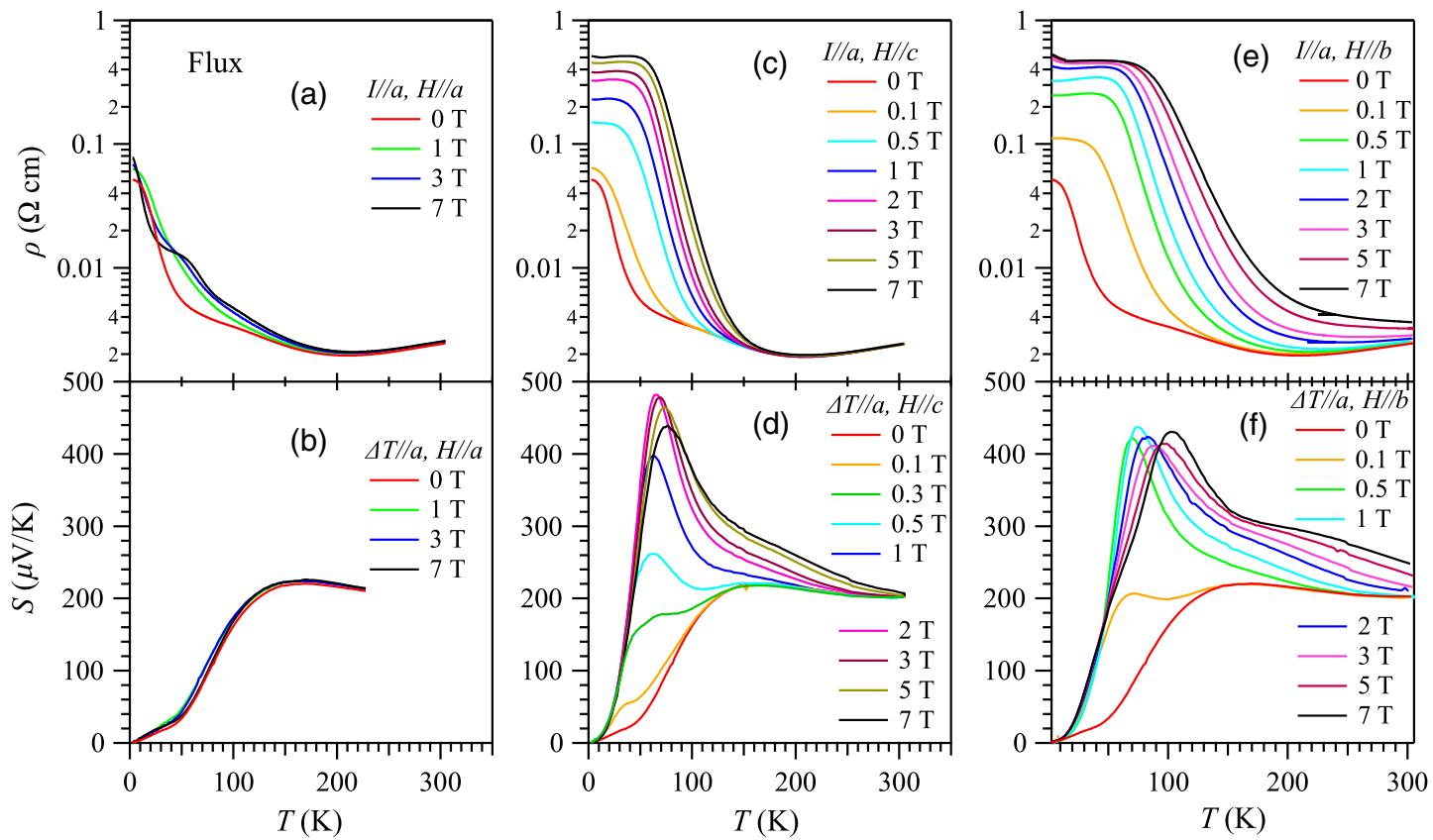

FIG. 4. Temperature dependence of resistivity $\rho(T)$ and thermopower $S(T)$ for the Flux sample with magnetic field applied along the three principal axes. 
and electronic structures, such an anisotropic magnetic field effect on the transport properties can be understood in terms of the electron cyclotron motion in the presence of magnetic fields; the effect is the strongest when the magnetic field is perpendicular to the current applied along the $a$ axis within the $\mathrm{ZrTe}_{5}$ sheets. Moreover, the largest Laudau level interval along the $b$ axis, i.e., $\Delta_{\mathrm{LL}}^{b} \gg \Delta_{\mathrm{LL}}^{c}>\Delta_{\mathrm{LL}}^{a}$, shows the influence of the magnetic field in a much more extended temperature range. These results thus suggest that the transport anomalies might originate from an intrinsic, common band structure of $\mathrm{ZrTe}_{5}$, as demonstrated by our first-principles calculations shown in Fig. 7.

\section{Structural and composition characterization}

In order to uncover the origin of distinct transport properties at zero field, we perform detailed characterizations on the crystal structure and chemical composition by means of single-crystal $\mathrm{x}$-ray diffraction (XRD) and energy dispersive spectroscopy (EDS) for the two kinds of single crystals. The single-crystal XRD data for both crystals can be well described by the orthorhombic (space group $\mathrm{Cmcm}$, No. 63) $\mathrm{ZrTe}_{5}$ structure having $\mathrm{Zr}$ and $\mathrm{Te} 1$ atoms at $4 c(0, y, 1 / 4)$ sites, and $\mathrm{Te} 2$ and $\mathrm{Te} 3$ atoms at $8 f(0$, $y, z)$ positions, respectively. The refinements converge well for both crystals, with the average and weighted reliability factors $R_{1}=0.0488(0.0267)$ and $w R_{1}=0.1337(0.0406)$ for CVT (Flux), respectively. The smaller $R$ factors of the Flux sample suggest a better crystal quality for those grown with the flux method described above. Detailed information about the structure refinements is given in Table I. The obtained atomic coordination, occupancies, and selected bond lengths and bond angles are listed in Tables S1 and S2, respectively, in Supplementary Material Ref. [27]. As seen in Table I, the obtained unit-cell parameters are $a=3.9830(3.9813) \AA, b=14.493(14.5053) \AA, c=$ 13.700(13.7030) $\AA$, and $V=790.8(791.35) \AA^{3}$ for CVT (Flux), respectively. These values are close to those reported previously [1,2], and their differences are less than $0.1 \%$. But a closer inspection shows that, unlike the $a$ axis, the $b$ and $c$ axes of the Flux sample are slightly longer than those of the CVT sample, resulting in a larger unit-cell volume for the Flux sample than the CVT sample. One of the important implications from the single-crystal XRD refinements is that the CVT crystal is slightly Te deficient, with an average composition $\mathrm{ZrTe}_{4.86}$, while the Flux crystal has a nearly perfect stoichiometry of $\mathrm{ZrTe}_{4.98}$. From the obtained site occupancies shown in Table S1 in Supplemental Material [27], the Te vacancies in CVT are located mainly at the $\mathrm{Te} 2$ and $\mathrm{Te} 3$ sites, about $3 \%-4 \%$.

The chemical compositions and the presence of $\mathrm{Te}$ deficiency in CVT are further verified via the EDS measurements on a number of crystals that are cleaved

TABLE I. Crystal data and structure refinement for $\mathrm{ZrTe}_{5}$ (CVT and Flux) single crystals.

\begin{tabular}{|c|c|c|}
\hline Empirical formula & $\mathrm{ZrTe}_{5}(\mathrm{CVT})$ & $\mathrm{ZrTe}_{5}$ (Flux) \\
\hline Crystal system, space group & Orthorhombic, $\mathrm{Cmcm}$ & Orthorhombic, $\mathrm{Cmcm}$ \\
\hline \multicolumn{3}{|l|}{ Unit cell dimensions } \\
\hline$a(\AA)$ & $3.9830(7)$ & $3.9813(3)$ \\
\hline$b(\AA)$ & $14.493(3)$ & $14.5053(14)$ \\
\hline$c(\AA)$ & $13.700(3)$ & $13.7030(13)$ \\
\hline Volume $\left(\AA^{3}\right), \mathrm{Z}$ & 790.8(3), 4 & $791.35(12), 4$ \\
\hline Density (calculated) $\left(\mathrm{g} / \mathrm{cm}^{3}\right)$ & 5.977 & 6.121 \\
\hline Formula weight & $711.67\left(\mathbf{Z r T e}_{\mathbf{4 . 8 6}}\right)$ & $729.22\left(\mathbf{Z r T e}_{\mathbf{5}}\right)$ \\
\hline Temperature $(\mathrm{K})$ & $273(2)$ & $273(2)$ \\
\hline Wavelength $(\AA)$ & 0.71073 & 0.71073 \\
\hline Absorption coefficient $\left(\mathrm{mm}^{-1}\right)$ & 18.859 & 19.344 \\
\hline $\mathrm{F}(000)$ & 1171.0 & 1200.0 \\
\hline Crystal size (mm) & $0.16 \times 0.21 \times 0.88$ & $0.14 \times 0.28 \times 0.61$ \\
\hline$\theta$ range for data collection $\left({ }^{\circ}\right)$ & $2.811-27.606$ & $2.809-27.444$ \\
\hline Limiting indices & $-5 \leq h \leq 5,-18 \leq k \leq 18-17 \leq l \leq 17$ & $-4 \leq h \leq 5,-18 \leq k \leq 18-17 \leq 1 \leq 17$ \\
\hline Reflections collected/unique & $4443 / 538$ & $4512 / 544$ \\
\hline $\mathrm{R}_{\mathrm{int}}$ & 0.0586 & 0.0504 \\
\hline Refinement method & Full-matrix least-squares on $F^{2}$ & Full-matrix least-squares on $F^{2}$ \\
\hline Data/restraints/parameters & $538 / 0 / 22$ & $539 / 0 / 22$ \\
\hline Goodness-of-fit on $F^{2}$ & 1.287 & 1.173 \\
\hline Final $\mathrm{R}$ indices $[I>2 \sigma(I)]$ & $R_{1}=0.0483, \mathrm{wR}_{2}=0.1335$ & $R_{1}=0.0215, \mathrm{wR}_{2}=0.0396$ \\
\hline $\mathrm{R}$ indices (all data) & $R_{1}=0.0488, \mathrm{wR}_{2}=0.1337$ & $R_{1}=0.0267, \mathrm{wR}_{2}=0.0406$ \\
\hline Largest different peak and hole $\left(e \AA^{-3}\right)$ & $3.021,-3.218$ & $1.055,-1.303$ \\
\hline
\end{tabular}


right before the measurements. The results are summarized in Table S3 in Supplemental Material [27]. The average $\mathrm{Zr}:$ Te molar ratios are estimated to be $1: 4.60 \pm 0.20$ and 1:4.98 \pm 0.17 for CVT and Flux, respectively. Although the data show a large variance, these results are, in general, consistent with the results of single-crystal XRD refinements; i.e., a considerable Te deficiency is present in the CVT crystal but is absent in the Flux crystals. Such a difference should be attributed to the different growth conditions for these two techniques. Although extra Te was added during the growth of CVT crystals, the evaporation of Te invariably produces a sizable Te deficiency; as a matter of fact, Te crystals were indeed observed after the CVT growth. In contrast, crystal growth in the Te self-flux using the CCS is more effective in achieving the stoichiometric composition. From the above characterizations, we conclude that the CVT crystals contain a considerable amount of Te deficiency, while the Flux samples are very close to the ideal stoichiometry.

\section{Carrier density and mobility}

Since the Te vacancies are an expected $n$-type dopant, evaluation of the carrier density on these two kinds of $\mathrm{ZrTe}_{5}$ crystals can provide further evidence to substantiate the above results. Figures 5(a) and 5(b) present the field dependences of longitudinal magnetoresistance $\mathrm{MR}=$ $[\rho(H) / \rho(0 \mathrm{~T})-1] \times 100 \%$ and Hall resistivity $\rho_{x y}$ for the CVT sample at various temperatures. The current was applied along the $a$ axis, and the magnetic field was oriented along the $b$ axis. Pronounced Shubnikov-de Haas ( $\mathrm{SdH})$ quantum oscillations are observed in MR at $T \leq 10 \mathrm{~K}$, and the MR reaches about $900 \%$ at $1.8 \mathrm{~K}$ and $5 \mathrm{~T}$, in agreement with the previous reports [14,30]. With increasing temperature, the $\mathrm{SdH}$ quantum oscillations smear out, and the magnitude of MR first decreases in the temperature range $1.8-100 \mathrm{~K}$ and then increases again up to $170 \mathrm{~K}$ as shown in Fig. 5(a). Here, $\rho_{x y}(H)$ exhibits a negative slope and changes slightly at $T<T_{P}=131 \mathrm{~K}$, signalling the dominant electron carriers at low temperatures, in accordance with the negative $S(T)$ shown in Fig. 2. The observation of an initial positive slope in the $\rho_{x y}(H)$ curve at $131 \mathrm{~K}$ indicates the appearance of high-mobility hole carriers around $T_{p}$, but the electron carriers with a large density remain at this temperature, given the large negative slope at higher fields. The positive slope of $\rho_{x y}(H)$ curves above $T_{p}$ signals the switch to dominant hole carries at high temperatures.
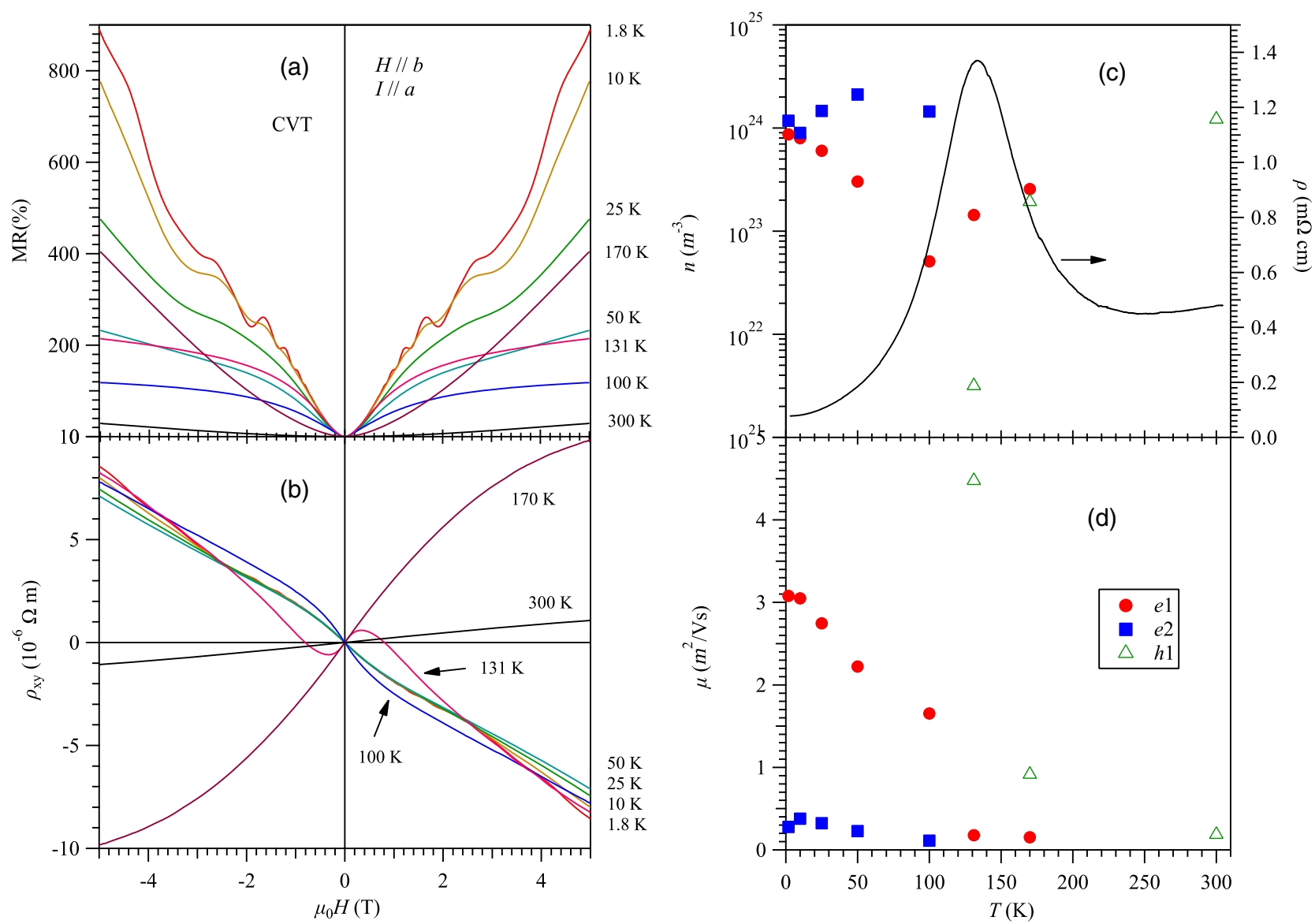

FIG. 5. Field dependences of (a) longitudinal magnetoresistance MR $=[\rho(H) / \rho(0 \mathrm{~T})-1] \times 100 \%$ and (b) Hall resistivity $\rho_{x y}$ for the CVT sample at various temperatures. Temperature dependence of the (c) carrier density $n$ and (d) mobility $\mu$ are extracted from the fitting to the Hall conductivity $\sigma_{x y}$. The resistivity data measured on the same sample are also shown in panel (c). 
In order to extract the quantitative information about the charge carriers, we fit the Hall conductivity, $\sigma_{x y}=$ $\rho_{x y} /\left(\rho_{x x}{ }^{2}+\rho_{x y}{ }^{2}\right)$, with the two-carrier model, viz.

$$
\sigma_{x y}=e B\left( \pm \frac{n_{1} \mu_{1}^{2}}{1+\mu_{1}^{2} B^{2}} \pm \frac{n_{2} \mu_{2}^{2}}{1+\mu_{2}^{2} B^{2}}\right)
$$

where $n_{1,2}$ and $\mu_{1,2}$ are the density and mobility for two types of charge carriers. In Eq. (1), $e>0$ is the elementary charge and $B$ is the magnetic flux density, respectively. The plus and minus signs should be taken into consideration when the corresponding carrier is a hole or an electron, respectively. All of the Hall conductivity data can be fitted well with the two-carrier model given in Eq. (1). The obtained carrier density $n$ and mobility $\mu$ are displayed in Fig. 5(c) and 5(d), in which the solid and open symbols represent the electron and hole carries, respectively. As can be seen, there are two kinds of electron carriers with similar density but different mobility at $T<T_{p}$, e.g., $n=$ $1.28 \times 10^{24} \mathrm{~m}^{-3}$ and $\mu=0.28 \mathrm{~m}^{2} / \mathrm{Vs}$ for $e 2$, and $n=$ $8.66 \times 10^{23} \mathrm{~m}^{-3}$ and $\mu=3.08 \mathrm{~m}^{2} / \mathrm{Vs}$ for $e 1$ at $1.8 \mathrm{~K}$. With increasing temperature, both $n$ and $\mu$ change slightly

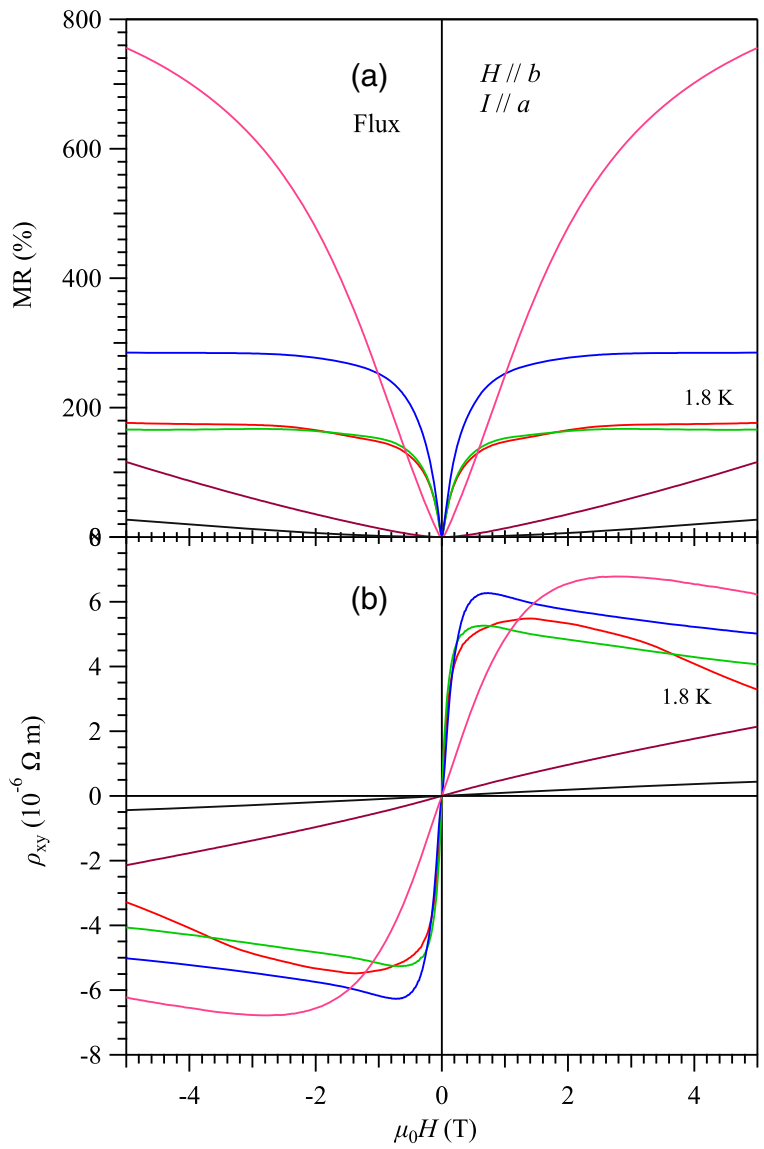

for $e 2$, whereas those of $e 1$ decrease quickly. At $T=T_{p}$, a high-mobility, low-density hole carrier coexists with a lowmobility, high-density electron carrier. The density of the hole carrier keeps increasing, but the mobility decreases. At $300 \mathrm{~K}$, the electrical transport properties can be best described by considering only one kind of hole carrier with $n=1.22 \times 10^{24} \mathrm{~m}^{-3}$ and $\mu=0.19 \mathrm{~m}^{2} / \mathrm{Vs}$, similar to that of $e 2$ at $1.8 \mathrm{~K}$. As superimposed in Fig. 5(a), the emergence of a resistivity peak is accompanied by the switch of one electron carrier to a hole carrier, or the Lifshitz transition as reported recently [31].

Similar measurements and data analysis were also performed for the Flux crystal, and the results are given in Fig. 6. As can be seen, the $\operatorname{MR}(H)$ shows a saturation behavior, and the largest $\mathrm{MR} \sim 760 \%$ at $1.8 \mathrm{~K}$ and $5 \mathrm{~T}$ occurs around $100 \mathrm{~K}$, in accordance with the data shown in Fig. 4. All $\rho_{x y}(H)$ curves have a positive initial slope in the whole temperature range, consistent with the positive $S(T)$ data shown in Fig. 2. The $\rho_{x y}(H)$ curves at $T \leq 100 \mathrm{~K}$ first exhibit a quick initial rise for $H<0.5 \mathrm{~T}$ and then show a bendover, implying the presence of two kinds of hole carriers. A similar two-carrier fitting was also applied. As shown in Figs. 6(c) and 6(d), there are indeed two kinds of

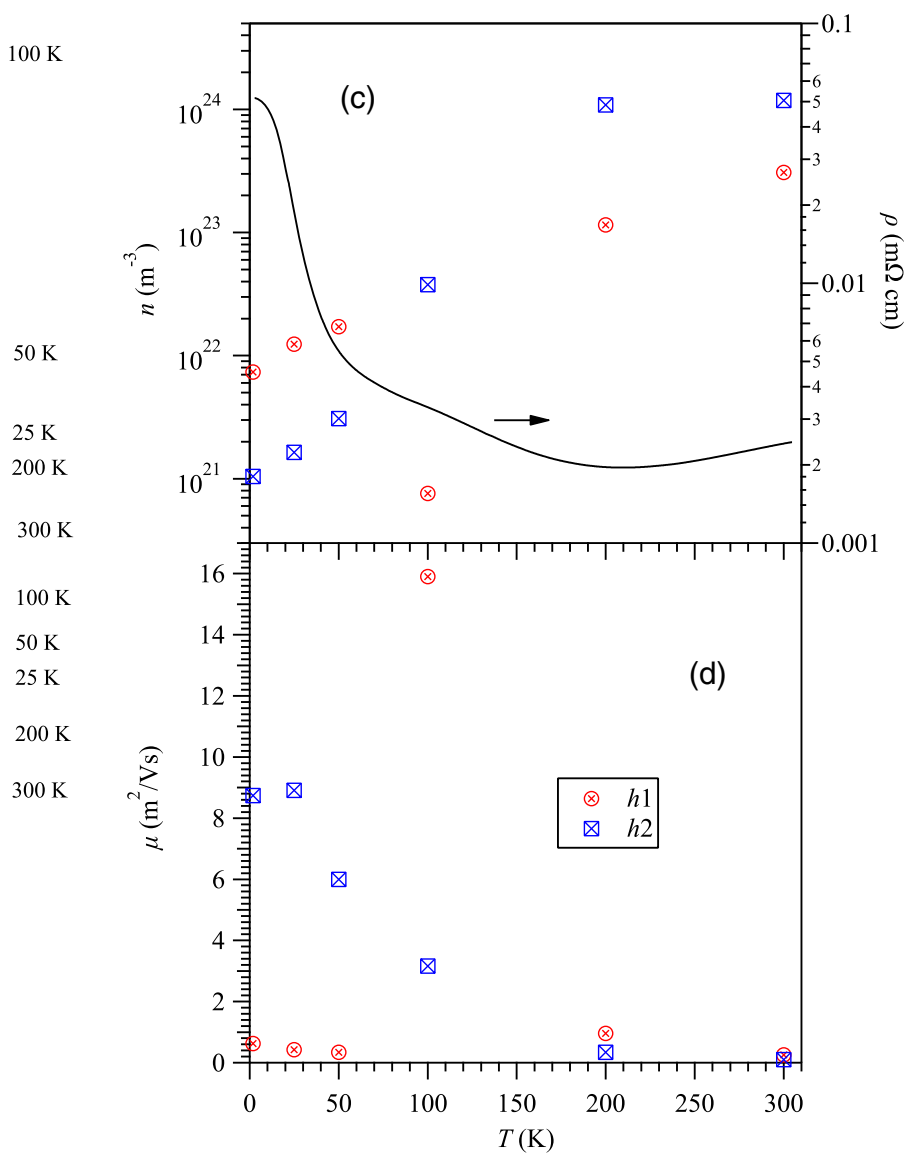

FIG. 6. Field dependences of (a) longitudinal magnetoresistance MR $=[\rho(H) / \rho(0 \mathrm{~T})-1] \times 100 \%$ and (b) Hall resistivity $\rho_{x y}$ for the Flux sample at various temperatures. Temperature dependence of the (c) carrier density $n$ and (d) mobility $\mu$ are extracted from the fitting to the Hall conductivity $\sigma_{x y}$. The resistivity data are also shown in panel (c). 
hole carriers in the whole temperature range. The carrier density and mobility are $n=7.36 \times 10^{21} \mathrm{~m}^{-3}$ and $\mu=$ $0.62 \mathrm{~m}^{2} / \mathrm{Vs}$ for $h 1$, and $n=1.04 \times 10^{21} \mathrm{~m}^{-3}$ and $\mu=$ $8.74 \mathrm{~m}^{2} / \mathrm{Vs}$ for $h 2$ at $1.8 \mathrm{~K}$. The carrier densities of the Flux sample are more than two orders lower than those of the CVT samples, in support of the scenario that the CVT sample contains more Te deficiency. With increasing temperature, the carrier densities increase steadily and reach $1.18 \times 10^{24} \mathrm{~m}^{-3}$ for $h 2$ and $3.08 \times 10^{23} \mathrm{~m}^{-3}$ for $h 1$, which are in the same level as those of the CVT samples. The mobility for the high-density hole is nearly unchanged with temperature, while that of the low-density hole decreases monotonically with temperature in a manner resembling the evolution of resistivity shown in Fig. 6(a). These characterizations demonstrate that the nearly stoichiometric $\mathrm{ZrTe}_{5}$ grown with the flux method has an extremely low carrier density, whose population increases with temperature as an intrinsic semiconductor. In contrast, the Te-deficient $\mathrm{ZrTe}_{5-\delta}$ crystals grown with the CVT technique are heavily electron doped, in agreement with the single-crystal XRD and EDS results shown above.

Based on the above, we can rationalize the distinct transport properties of $\mathrm{ZrTe}_{5}$ shown in Fig. 2 in terms of the different chemical compositions. The transport properties of the Flux sample should be regarded as the intrinsic, or very close to the intrinsic, behaviors of $\mathrm{ZrTe}_{5}$; the observed semiconducting-like $\rho(T)$ is consistent with the firstprinciples band structure calculations [11], showing a finite gap near the Fermi energy, as also detected by the scanning tunneling microscopy measurement [17]. The positive $S(T)$ in the whole temperature range indicates that the Fermi level is slightly below the top of the valence band. On the other hand, the presence of a considerable amount of Te deficiency in the CVT sample introduces electron carriers to the conduction band, giving rise to a metallic resistivity and a negative thermopower at low temperatures, as observed. As will be shown below, the resistivity peak and the thermopower sign reversal at $T_{p}$ can be reproduced by our parameter-free transport calculations when taking into account the intrinsic band structure anisotropies at the valence band maximum and conduction band minimum. Our results also provide a simple physical explanation for the contradictory reports in the literature regarding the electronic structure of $\mathrm{ZrTe}_{5}$, i.e., semiconductor versus Dirac semimetal [12-18].

\section{E. Electronic structure and transport calculations}

Density functional calculations were done for $\mathrm{ZrTe}_{5}$ using the general potential LAPW method [20] as implemented in the WIEN2k code [21]. The density of states shows hybridized bands derived from Te $p$ and $\mathrm{Zr} d$ states. This is similar to prior reports [11]. The Te contributions are dominant, as may be expected from the stoichiometry, and this is also the case near the Fermi levels. The $\mathrm{Zr}$ $d$ states are more prominent in the conduction bands.
The band structure formation is a consequence of Te $p$ states, which make the spin-orbit interaction particularly important. The complexity of the crystal structure, Fig. 1(a), can then be readily understood as due to the competition between Te $p$ bonding and accommodation of the metal ions, similar to other complex structure tellurides, such as $\mathrm{IrTe}_{2}$ [32]. We note that the separation of the metal ions is $4 \AA$, implying that direct interactions between cations will be weak and that their coupling will be via the Te lattice.

What is important for transport is the electronic structure near the Fermi energy. We found that $\mathrm{ZrTe}_{5}$ is a semiconductor with an indirect band gap of $0.073 \mathrm{eV}$. The density of states near the gap is shown in Fig. 7(a). It is important to note that unlike most topological systems, the bands near the valence band maximum (VBM) are very different from those near the conduction band minimum (CBM). In particular, the onset of the density of states is much steeper for electrons at the CBM than for holes at the VBM. The band shapes at the VBM and CBM are also very different. This is seen in Fig. 7(b), which depicts the isosurfaces of the highest valence band $0.05 \mathrm{eV}$ below the VBM and the lowest conduction band $0.05 \mathrm{eV}$ above the CBM. This is also seen in the band structure shown in Fig. 7(c) [the Brillouin zone path is given in Fig. 7(d)]. There is an inverted band structure at the zone center, with similar electron and hole shapes, but importantly, there are other bands near the band edge, which are very different for electrons and holes. In addition to the pockets near the zone center, the conduction bands show additional pockets in the middle of the zone and at the zone boundaries. Thus, it is clearly seen that the shapes of the hole and electron pockets overall are different; therefore, one may anticipate different transport properties. This idea relates to the thermoelectric properties as well. In topological insulators, a spin-orbitinduced gap opening generally leads to similar character and related complex band shapes at the valence and conduction bands, which has been shown to be important in producing band complexity and, when other conditions are met, high thermoelectric performance [33,34]. Here, the complex-shaped bands are present, as expected. However, bipolar conduction, which is important at temperatures at and above the resistivity peak for $\mathrm{ZrTe}_{5}$, as discussed below, is extremely detrimental to thermoelectric performance as it reduces the thermopower and greatly increases the electronic thermal conductivity.

Figure 8 shows calculated values of the transport function $\sigma / \tau$, where $\sigma$ is conductivity and $\tau$ is the unknown inverse scattering rate; the ratio is determined by the band structure alone and is proportional to the square of the optical Drude plasma frequency, as obtained from the electronic structure for a temperature of $50 \mathrm{~K}$, using the BoltzTraP code. [25]. The conductivity along the $b$-axis direction $(y)$ is very low, reflecting the fact that from an electronic transport point of view, the material is highly two dimensional. There is also significant anisotropy in the $a c$ 
(a)

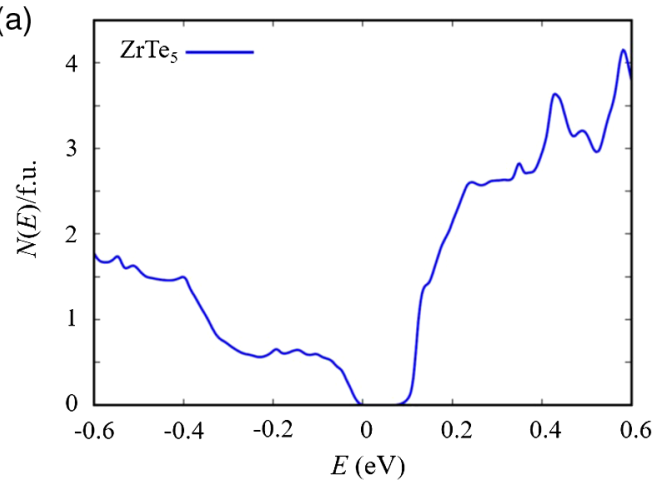

(b)

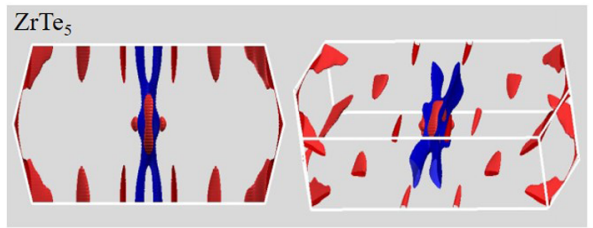

(c)

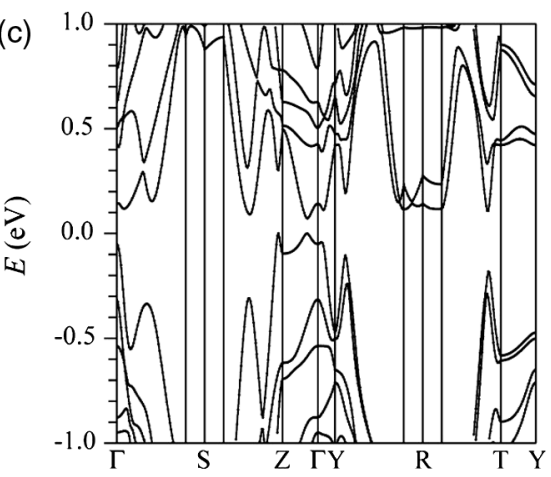

(d)

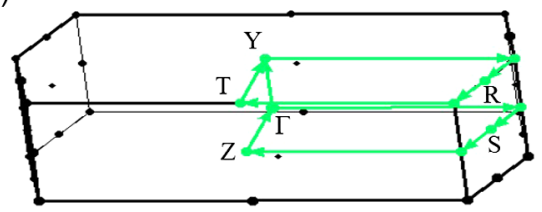

FIG. 7. (a) Total electronic density of states (DOS) of $\mathrm{ZrTe}_{5}$ on a per-formula unit basis. Note the asymmetry between electrons and holes. (b) Band energy isosurfaces $0.05 \mathrm{eV}$ from the band edges for $\mathrm{ZrTe}_{5}$. The $\Gamma$ point is at the center of the depicted zones, while the $k_{x}$ direction is along the horizontal axis for the left panel, while the right panel shows a tilted view. Hole surfaces below the valence band maximum are in blue, while electron surfaces above the conduction band minimum are in red. Note the very different structure of the hole and electron sheets. (c) Band structure of $\mathrm{ZrTe}_{5}$; note the semiconducting character. (d) Brillouin zone path for the band structure.

plane, consistent with the report of Tritt and co-workers [26]. Importantly, the high-conductivity directions for electrons and holes are opposite, and the hole conductivity is higher than the electron conductivity for transport along the usually measured $a$ axis $(x)$. Specifically, the hole conductivity is highest along $a$, while the electron conductivity is highest along $c$. This is in accordance with transport data for few-layer, $p$-type $\mathrm{ZrTe}_{5}$ [35], where a hole mobility approximately 2 times larger was measured along the $a$ axis compared with the $c$ axis. This has important implications.

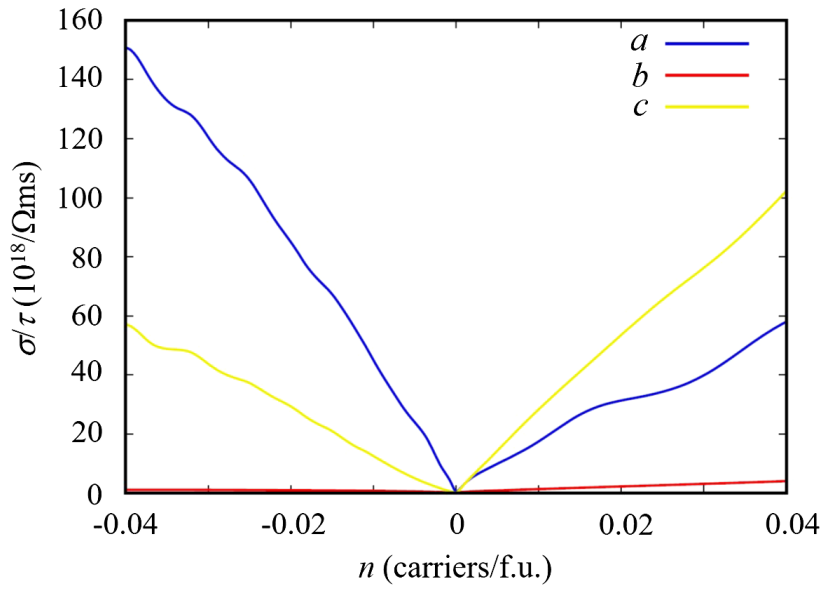

FIG. 8. Transport function $\sigma / \tau$ along the three crystallographic directions for $\mathrm{ZrTe}_{5}$.
Specifically, $\mathrm{ZrTe}_{5}$ is usually $n$ type at low temperatures because of the Te deficiency, as verified in the present work. For a heavily doped narrow-gap system, one expects the behavior of a degenerate doped (i.e., low carrier density metallic) system up to some temperature at which point bipolar conduction starts. At this point, the carrier density will increase, keeping a balance between electrons and holes, and at high temperatures, $S(T)$ will take the sign of the higher conductivity carriers. One may also anticipate that the resistivity will increase with temperature because of electron phonon scattering below the crossover temperature where bipolar conduction becomes important; it will then decrease because of the increase in carrier density. Strong $T$ dependence could occur near this crossover because of the exponential factors that appear in the Fermi function. This picture is confirmed by direct calculations of the transport functions.

Figure 9(a) shows the calculated $S(T)$ along the $a$ axis for different $p$ - and $n$-type doping levels in $\mathrm{ZrTe}_{5}$ (corresponding data for $\mathrm{HfTe}_{5}$ in comparison with $\mathrm{ZrTe}_{5}$ are shown in Fig. S3 of the Supplemental Material [27]). These values were obtained in the standard constant scattering time approximation, setting the hole and electron scattering rates equal. As seen, for $n$-type doping levels, $\mathrm{ZrTe}_{5}$ displays a strong doping-dependent crossover from electronlike to holelike $S(T)$ and a relatively constant value at high temperature, as we observed experimentally in Fig. 2. The crossover temperature for $\mathrm{HfTe}_{5}$ is lower than that of $\mathrm{ZrTe}_{5}$ (Fig. S3 in Supplemental Material [27]), consistent with experimental reports. This difference is due to the 

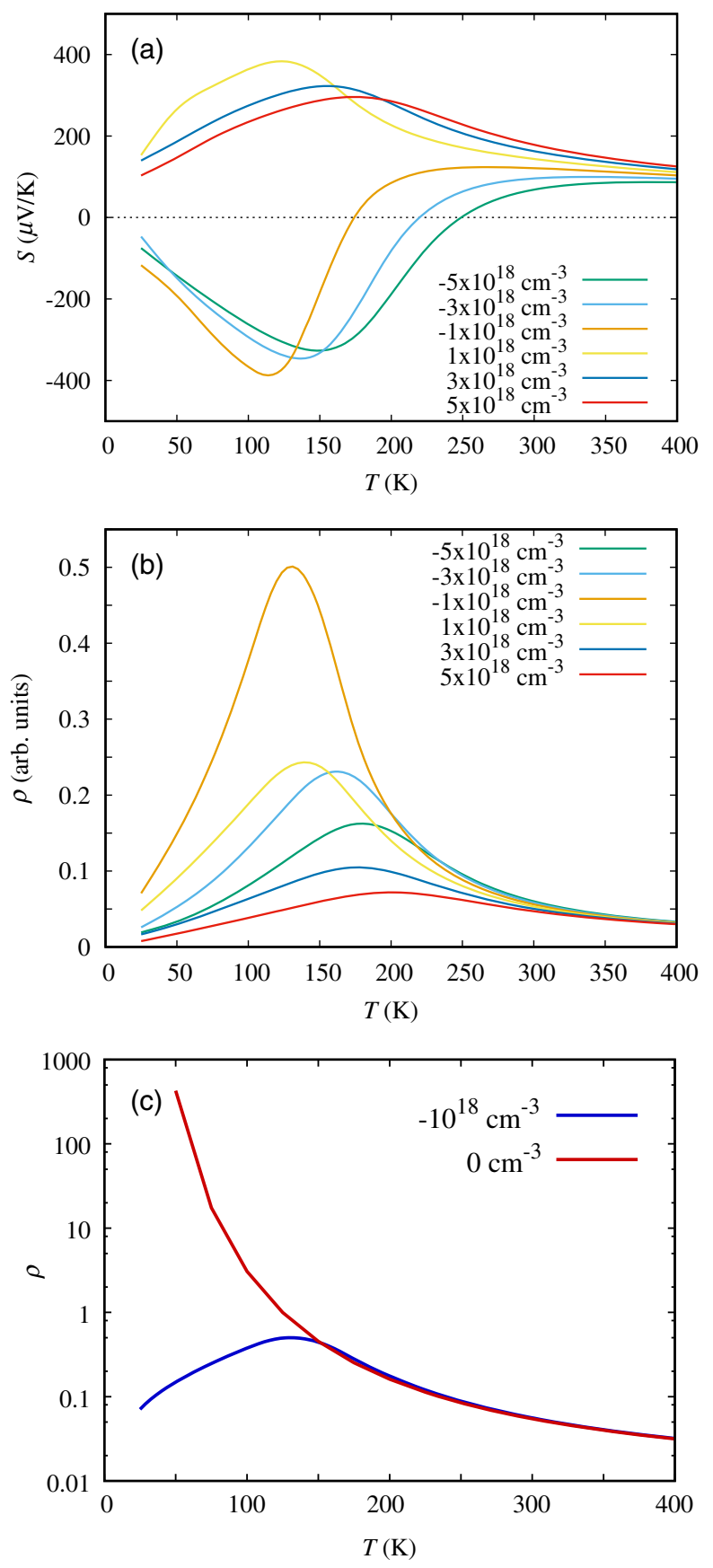

FIG. 9. (a) The $a$-axis $S(T)$ for various doping levels as obtained in the constant-scattering-time approximation for $\mathrm{ZrTe}_{5}$. (b) The $a$-axis resistivity transport function, $\rho$ (see text) for $\mathrm{ZrTe}_{5}$. (c) The $a$-axis resistivity transport function on a log scale comparing $n$-type doped and undoped material.

smaller band gap of $\mathrm{HfTe}_{5}$ [Fig. S2(a) in Supplemental Material [27] ]. We emphasize that we have not introduced any adjustable parameter to obtain these results. Turning to the value of the $p$-type thermopower at high temperature, it can be seen that our values are somewhat lower than typical experimental values. Such a difference could be corrected by assuming different scattering rates $\tau$ for electrons and holes.
While this may be reasonable considering the different nature of the electron and hole bands, it would introduce more parameters in the calculations.

For the resistivity, we consider the simplest model that follows from the above considerations. Specifically, we take $\sigma / \tau$ as obtained directly from the band structure and adopt a metallic electron-phonon dependence $\tau$ proportional to $1 / T$ $[36,37]$. The inverse of this function is plotted for various doping levels in Fig. 9(b). As can be seen, there are strong peaks in resistivity for low $n$-type doping levels $\left(10^{24} \mathrm{~m}^{-3}\right)$ at temperatures consistent with experiment for $\mathrm{ZrTe}_{5}$. Data for $\mathrm{HfTe}_{5}$ (Fig. S3 in Supplemental Material [27]) show a similar peak but at lower temperatures than that for $\mathrm{ZrTe}_{5}$. We also note that there is a doping-level dependence that explains the sample-to-sample variation in these materials. The fact that the transport, even for $n$-type samples, is strongly affected by both holes and electrons at and above the resistivity peak, and that these involve the conductivities in the $a$-axis and $c$-axis directions, but not significantly along $b$, is consistent with the field orientation dependences, specifically the lack of enhancement in the transition only when the field is along the $a$ axis. Importantly, a semiconducting behavior, as seen in Fig. 2(a) for the Flux sample, can be reproduced for the undoped material as shown in Fig. 9(c). Thus, standard transport theory applied to the band structure calculated using standard methods can reproduce the resistivity peak and behavior of the thermopower for $\mathrm{ZrTe}_{5}$ without adjustable parameters. Moreover, this same theory applied in the same way also reproduces the transport properties of $\mathrm{HfTe}_{5}$ including the lower temperature anomaly for this material.

Finally, we make a brief comment on thermopower. Normally, thermopower is the most isotropic among the electrical transport quantities, particularly in semiconductors. However, open Fermi surfaces can lead to strongly anisotropic thermopower as in the case of $\mathrm{PdCoO}_{2}$ [38]. As seen in Fig. 7(b), the energy isosurfaces become open at low energies. Therefore, it is of interest to examine the transport behavior for the other conduction directions, along $c$ (note that there is very little conduction along $b$ ). We predict that, in this direction, the behavior of the thermopower is opposite to that along $a$. Specifically, there is a change in sign for $p$-type materials, and the high temperature saturation for both $n$ - and $p$-type samples is to a negative thermopower. The absolute values are larger for $n$-type than for $p$-type samples because of the heavier bands, as seen in the stronger density-of-states onset for the conduction bands. The opposite behavior of $S(T)$ along $a$ and $c$ is a consequence of the different high-conductivity directions for electrons and holes. It will be of interest to test this prediction should suitable samples become available.

In the thermoelectric application, the onset of bipolar conduction is invariably highly detrimental to the figure of merit $Z T \equiv S^{2} \sigma T / \kappa$, where $S, \sigma$, and $\kappa$ stand for thermopower, electrical conductivity, and thermal conductivity, 
respectively. This leads to a strong enhancement of $\kappa$ and a decrease of $S$. Thus, for any application, it will be important to avoid the bipolar regime. This is defined by the resistivity maximum. Therefore, the best performance would be at cryogenic temperatures below the resistivity maximum, and according to our results, for the thermopower, it would likely be best along the $c$ axis. It is noteworthy that the predicted values of $S$ for the naturally occurring $n$-type sample at $150 \mathrm{~K}$, for carrier concentrations as high as $5 \times 10^{24} \mathrm{~m}^{-3}$, exceed $300 \mu \mathrm{V} / \mathrm{K}$. Furthermore, the complex band shapes that arise from the spin-orbit splitting [11] are generally favorable for thermoelectric performance [33]. A key issue will be the extent to which the $n$-type doping level can be controlled to achieve appropriate $S$ and $\sigma$ in the temperature range below the onset of bipolar conduction.

\section{CONCLUSION}

In conclusion, we have verified experimentally that $\mathrm{ZrTe}_{5}$ single crystals with Te deficiency show interesting transport properties, including the resistance peak and the thermopower sign reversal around $T_{p}=135 \mathrm{~K}$. In contrast, the nearly stoichiometric $\mathrm{ZrTe}_{5}$ single crystals show $p$-type semiconducting transport behavior at low temperatures. Aided by the first-principles calculations, we have identified a common origin in the peculiar band structures of pentatellurides for these distinct transport behaviors. The long-standing puzzle of the transition in pentatellurides can be understood in terms of an onset of bipolar conduction for narrow-gap semiconductors, with bands having different anisotropies at the VBM and CBM. Our work demonstrates that the interesting properties of $\mathrm{ZrTe}_{5}$ can be effectively tailored by carefully controlling the Te content or band filling.

\section{ACKNOWLEDGMENTS}

The authors thank Dr. Hongming Weng, Dr. Zhong Wang, and Dr. Jian Wang for enlightening discussions. We are also grateful to Dr. Youting Song for his assistant in the single-crystal x-ray diffraction measurements. This work is supported by the National Key R\&D Program of China (Grants No. 2018YFA0305700 and No. 2014CB921500), the National Science Foundation of China (Grant No. 11574377), the Key Research Program of Frontier Sciences, and the Strategic Priority Research Program of the Chinese Academy of Sciences (Grants No. QYZDB-SSWSLH013 and No. XDB07020100). D. S. is supported by the Department of Energy, Basic Energy Sciences, through the MAGICS Center, Award No. DE-SC0014607. J.-Q. Y. is supported by the U.S. Department of Energy, Office of Science, Basic Energy Sciences, Materials Sciences and Engineering Division. D. G. M. acknowledges support from the Gordon and Betty Moore Foundation's EPiQS Initiative through Grant No. GBMF4416.
[1] S. Furuseth, L. Brattas, and A. Kjekshus, The Crystal Structure of $\mathrm{HfTe}_{5}$, Acta Chem. Scand. 27, 2367 (1973).

[2] H. Fjellavg and A. Kjekshus, Structural Properties of $\mathrm{ZrTe}_{5}$ and $\mathrm{HfTe}_{5}$ as Seen by Powder Diffraction, Solid State Commun. 60, 91 (1986).

[3] F. J. DiSalvo, R. M. Fleming, and J. V. Waszczak, Possible Phase Transition in the Quasi-One-Dimensional Materials $\mathrm{ZrTe}_{5}$ or $\mathrm{HfTe}_{5}$, Phys. Rev. B 24, 2935 (1981).

[4] S. Okada, T. Sambongi, M. Ido, Y. Tazuke, R. Aoki, and O. Fujita, Negative Evidences for Charge/Spin Density Wave in $\mathrm{ZrTe}_{5}$, J. Phys. Soc. Jpn. 51, 460 (1982).

[5] R. T. Littleton IV, T. M. Tritt, J. W. Kolis, and D. R. Ketchum, Transition-Metal Pentatellurides as Potential Low-Temperature Thermoelectric Refrigeration Materials, Phys. Rev. B 60, 13453 (1999).

[6] R. Y. Chen, S. J. Zhang, J. A. Schneeloch, C. Zhang, Q. Li, G. D. Gu, and N. L. Wang, Optical Spectroscopy Study of the Three-Dimensional Dirac Semimetal $\mathrm{ZrTe}_{5}$, Phys. Rev. B 92, 075107 (2015).

[7] M. Rubinstein, $\mathrm{HfTe}_{5}$ and $\mathrm{ZrTe}_{5}$ : Possible Polaronic Conductors, Phys. Rev. B 60, 1627 (1999).

[8] D. N. McIlroy, S. Moore, D. Q. Zhang, J. Wharton, B. Kempton, R. Littleton, M. Wilson, T. M. Tritt, and C. G. Olson, Observation of a Semimetal-Semiconductor Phase Transition in the Intermetallic $\mathrm{ZrTe}_{5}$, J. Phys. Condens. Matter 16, L359 (2004).

[9] G. Manzoni, A. Sterzi, A. Crepaldi, M. Diego, F. Cilento, M. Zacchigna, P. Bugnon, H. Berger, A. Magrez, M. Grioni, and F. Parmigiani, Ultrafast Optical Control of the Electronic Properties of $\mathrm{ZrTe}_{5}$, Phys. Rev. Lett. 115, 207402 (2015).

[10] Y. Zhang, C. Wang, L. Yu, G. Liu, A. Liang, J. Huang, S. Nie, X. Sun, Y. Zhang, B. Shen, J. Liu, H. Weng, L. Zhao, G. Chen, X. Jia, C. Hu, Y. Ding, W. Zhang, Q. Cao, C. Li et al., Electronic Evidence of Temperature-Induced Lifshitz Transition and Topological Nature in $\mathrm{ZrTe}_{5}$, Nat. Commun. 8, 15512 (2017).

[11] H. Weng, X. Dai, and Z. Fang, Transition-Metal Pentatelluride $\mathrm{ZrTe}_{5}$ and $\mathrm{HfTe}_{5}$ : A Paradigm for Large-Gap Quantum Spin Hall Insulators, Phys. Rev. X 4, 011002 (2014).

[12] Q. Li, D. E. Kharzeev, C. Zhang, Y. Huang, I. Pletikosic, A. V. Fedorov, R. D. Zhong, J. A. Schneeloch, G. D. Gu, and T. Valla, Chiral Magnetic Effect in $\mathrm{ZrTe}_{5}$, Nat. Phys. 12, 550 (2016).

[13] R. Y. Chen, Z. G. Chen, X.-Y. Song, J. A. Schneeloch, G. D. Gu, F. Wang, and N. L. Wang, Magnetoinfrared Spectroscopy of Landau Levels and Zeeman Splitting of ThreeDimensional Massless Dirac Fermions in $\mathrm{ZrTe}_{5}$, Phys. Rev. Lett. 115, 176404 (2015).

[14] G. Zheng, J. Lu, X. Zhu, W. Ning, Y. Han, H. Zhang, J. Zhang, C. Xi, J. Yang, H. Du, K. Yang, Y. Zhang, and M. Tian, Transport Evidence for the Three-Dimensional Dirac Semimetal Phase in $\mathrm{ZrTe}_{5}$, Phys. Rev. B 93, 115414 (2016).

[15] L. Moreschini, J. C. Johannsen, H. Berger, J. Denlinger, C. Jozwiak, E. Rotenberg, K. S. Kim, A. Bostwick, and M. Grioni, Nature and Topology of the Low-Energy States in $\mathrm{ZrTe}_{5}$, Phys. Rev. B 94, 081101(R) (2016).

[16] R. Wu, J.-Z. Ma, S.-M. Nie, L.-X. Zhao, X. Huang, J.-X. Yin, B.-B. Fu, P. Richard, G.-F. Chen, Z. Fang, X. Dai, 
H.-M. Weng, T. Qian, H. Ding, and S. H. Pan, Evidence for Topological Edge States in a Large Energy Gap near the Step Edges on the Surface of $\mathrm{ZrTe}_{5}$, Phys. Rev. X 6, 021017 (2016).

[17] X. B. Li, W. K. Huang, Y. Y. Lv, K. W. Zhang, C. L. Yang, B. B. Zhang, Y. B. Chen, S.-H. Yao, J. Zhou, M. H. Lu, L. Sheng, S. C. Li, J. F. Jia, Q.-K. Xue, Y. F. Chen, and D. Y. Xing, Experimental Observation of Topological Edge States at the Surface Step Edge of the Topological Insulator $\mathrm{ZrTe}_{5}$, Phys. Rev. Lett. 116, 176803 (2016).

[18] H. Wang, C.-K. Li, H. Liu, J. Yan, J. Wang, J. Liu, Z. Lin, Y. Li, Y. Wang, L. Li, D. Mandrus, X. C. Xie, J. Feng, and J. Wang, Chiral Anomaly and Ultrahigh Mobility in Crystalline $\mathrm{HfTe}_{5}$, Phys. Rev. B 93, 165127 (2016).

[19] P. C. Canfield, T. Kong, U. S. Kaluarachchi, and N. H. Jo, Use of Frit-Disc Crucibles for Routine and Exploratory Solution Growth of Single Crystalline Samples, Philos. Mag. 96, 84 (2016).

[20] D. J. Singh and L. Nordstrom, Planewaves Pseudopotentials and the LAPW Method, 2nd ed. (Springer, Berlin, 2006).

[21] P. Blaha, K. Schwarz, G. K. H. Madsen, D. Kvasnicka, and J. Luitz, Wien2k, An Augmented Plane Wave+Local Orbital Program for Calculating Crystal Properties (TU Vienna, Vienna, 2001).

[22] D. Singh, Ground-State Properties of Lanthanum: Treatment of Extended-Core States, Phys. Rev. B 43, 6388 (1991).

[23] E. Sjostedt, L. Nordstrom, and D. J. Singh, An Alternative Way of Linearizing the Augmented Plane-Wave Method, Solid State Commun. 114, 15 (2000).

[24] J. P. Perdew, K. Burke, and M. Ernzerhof, Generalized Gradient Approximation Made Simple, Phys. Rev. Lett. 77, 3865 (1996).

[25] G. K. H. Madsen and D. J. Singh, BoltzTraP. A Code for Calculating Band-Structure Dependent Quantities, Comput. Phys. Commun. 175, 67 (2006).

[26] T. M. Tritt, N. D. Lowhorn, R. T. Littleton IV, A. Pope, C. R. Feger, and J. W. Kolis, Large Enhancement of the Resistivity Anomaly in the Pentatelluride Materials $\mathrm{HfTe}_{5}$ and $\mathrm{ZrTe}_{5}$ with Applied Magnetic Field, Phys. Rev. B 60, 7816 (1999).

[27] See Supplemental Material at http://link.aps.org/ supplemental/10.1103/PhysRevX.8.021055 for the structural information, results of EDS, and magnetoresistance of $\mathrm{ZrTe}_{5}$ single crystals, as well as the calculated total density of states and transport properties of $\mathrm{ZrTe}_{5}$ and $\mathrm{HfTe}_{5}$ for comparison.

[28] Y. Li, Z. Wang, P. Li, X. Yang, Z. Shen, F. Sheng, X. Li, Y. $\mathrm{Lu}$, Y. Zheng, and Z. Xu, Negative Magnetoresistance in Weyl Semimetals NbAs and NbP: Intrinsic Chiral Anomaly and Extrinsic Effects, Front. Phys. 12, 127205 (2017).

[29] P. Goswami, J. H. Pixley, and S. Das Sarma, Axial Anomaly and Longitudinal Magnetoresistance of a Generic ThreeDimensional Metal, Phys. Rev. B 92, 075205 (2015).

[30] X. Yuan, C. Zhang, Y. Liu, A. Narayan, C. Song, S. Shen, X. Sui, J. Xu, H. Yu, Z. An, J. Zhao, S. Sanvito, H. Yan, and F. Xiu, Observation of Quasi-Two-Dimensional Dirac Fermions in $\mathrm{ZrTe}_{5}$, NPG Asia Mater. 8, e325 (2016).

[31] H. Chi, C. Zhang, G. D. Gu, D. E. Kharzeev, X. Dai, and Q. Li, Lifshitz Transition Mediated Electronic Transport Anomaly in Bulk ZrTe ${ }_{5}$, New J. Phys. 19, 015005 (2017).

[32] Q. Li, W. Lin, J. Yan, X. Chen, A. G. Gianfrancesco, D. J. Singh, D. Mandrus, S. V. Kalinin, and M. Pan, Bond Competition and Phase Evolution on the $\mathrm{IrTe}_{2}$ Surface, Nat. Commun. 5, 5358 (2014).

[33] H. Shi, D. Parker, M.-H. Du, and D. J. Singh, Connecting Thermoelectric Performance and Topological-Insulator Behavior: $\mathrm{Bi}_{2} \mathrm{Te}_{3}$, and $\mathrm{Bi}_{2} \mathrm{Te}_{2} \mathrm{Se}$ from First Principles, Phys. Rev. Applied 3, 014004 (2015).

[34] G. Z. Xing, J. F. Sun, Y. W. Li, X. F. Fan, W. T. Zheng, and D. J. Singh, Electronic Fitness Function for Screening Semiconductors as Thermoelectric Materials, Phys. Rev. Mater. 1, 065405 (2017).

[35] G. Qiu, Y. Du, A. Charnas, H. Zhou, S. Jin, Z. Luo, D. Y. Zemlyanov, X. Xu, G. J. Cheng, and P. D. Ye, Observation of Optical and Electrical in-Plane Anisotropy in HighMobility Few-Layer ZrTe ${ }_{5}$, Nano Lett. 16, 7364 (2016).

[36] P. B. Allen, Empirical Electron-Phonon $\lambda$ Values from Resistivity of Cubic Metallic Elements, Phys. Rev. B 36, 2920 (1987).

[37] P. B. Allen, W. E. Pickett, and H. Krakauer, Anisotropic Normal-State Transport Properties Predicted and Analyzed for High-T $T_{c}$ Oxide Superconductors, Phys. Rev. B 37, 7482 (1988).

[38] K. P. Ong, D. J. Singh, and P. Wu, Unusual Transport and Strongly Anisotropic Thermopower in $\mathrm{PtCoO}_{2}$ and $\mathrm{PdCoO}_{2}$, Phys. Rev. Lett. 104, 176601 (2010). 